\title{
Enhancement of Planning Ability by Transcranial Direct Current Stimulation
}

\author{
Colleen A. Dockery, ${ }^{1,2}$ Ruth Hueckel-Weng, ${ }^{2}$ Niels Birbaumer, ${ }^{2,4}$ and Christian Plewnia ${ }^{3}$ \\ ${ }^{1}$ Max Planck Graduate School of Neural \& Behavioral Sciences and ${ }^{2}$ Institute of Medical Psychology \& Behavioral Neurobiology, University of Tuebingen, \\ D-72074 Tuebingen, Germany, ${ }^{3}$ Department of Psychiatry and Psychotherapy, University of Tuebingen, D-72076 Tuebingen, Germany, and ${ }^{4}$ Ospedale San \\ Camillo, Istituto di Ricovero e Cura a Carattere Scientifico, 30126 Venezia Lido, Italy
}

The functional neuroanatomy of executive function critically involves the dorsolateral prefrontal cortex. Transcranial direct current stimulation (tDCS) has been established as a noninvasive tool for transient modulation of cortical function. Here, we examined the effects of $\mathrm{tDCS}$ of the left dorsolateral prefrontal cortex on planning function by using the Tower of London task to evaluate performance during and after anodal, cathodal ( $1 \mathrm{~mA}, 15 \mathrm{~min}$ ), and sham $\mathrm{tDCS}$ in 24 healthy volunteers. The key finding was a double dissociation of polarity and training phase: improved performance was found with cathodal tDCS applied during acquisition and early consolidation, when preceding anodal tDCS, but not in the later training session. In contrast, anodal tDCS enhanced performance when applied in the later sessions following cathodal tDCS. Our results indicate that both anodal and cathodal tDCS can improve planning performance as quantified by the Tower of London test. Most importantly, these data demonstrate training-phase-specific effects of tDCS. We propose that excitability decreasing cathodal tDCS mediates its early beneficial effect through noise reduction of neuronal activity, whereas a further adaptive configuration of specific neuronal connections is supported by excitability enhancing anodal tDCS in the later training phase by enhanced efficacy of active connections. This gain of function was sustained in a follow-up 6 and 12 months after training. In conclusion, the specific coupling of stimulation and training phase interventions may support the treatment of cognitive disorders involving frontal lobe functions.

\section{Introduction}

Patients with frontal lobe pathology, such as schizophrenia, depression, and lesions, commonly express executive function impairment. Planning abilities are prerequisites for successful problem solving and effective behavior. The Tower of London (TOL) is a widely used test of planning ability (Shallice, 1982), and is established as a valid measure for cognitive skill acquisition (Peretti et al., 2002). Patient performance, particularly at higher levels of task load, differs from healthy controls and relates to findings in neuroimaging studies concerning the dorsolateral prefrontal cortex (DLPFC) as the most critical structure for solving the TOL task (Baker et al., 1996; Owen, 1997; Dagher et al., 1999; Rainville et al., 2002; Lazeron et al., 2004; Rasser et al., 2005; van den Heuvel et al., 2005). Left prefrontal activation has been associated with higher task demand in working memory (Olesen et al., 2004, Walter et al., 2007a,b) and in TOL problems (Morris et al., 1993; Owen et al., 1996; Rowe et al., 2001; Schall et al., 2003; van den

Received Jan. 6, 2009; revised April 30, 2009; accepted May 5, 2009.

This research was supported by a research and study scholarship for C.A.D. from the Deutscher Akademischer Austausch Dienst. N.B. is funded by the Deutsche Forschungsgemeinschaft (German Research Foundation), Bundesministerium für Bildung und Forschung and the Bernstein Center for Computational Neuroscience. C.P. was funded by the German Research Council Collaborative Research Center (SFB 550-A13). We thank Petr Cempirek for the illustrations, Ken Nash for technical support with the figures, and Tilman Gaber for statistical support.

Correspondence should be addressed to Colleen A. Dockery, Institute of Medical Psychology \& Behavioral Neurobiology, University of Tuebingen, Gartenstrasse 29, D-72074 Tuebingen, Germany. E-mail: colleen.dockery@ student.uni-tuebingen.de.

DOI:10.1523/JNEUROSCI.0065-09.2009

Copyright $\odot 2009$ Society for Neuroscience $\quad 0270-6474 / 09 / 297271-07 \$ 15.00 / 0$
Heuvel et al., 2003), and with superior or successful performance on the TOL task (Cazalis et al., 2003, 2006).

Transcranial direct current stimulation (tDCS) decreases cortical excitability with cathodal or increases excitability with anodal currents (Nitsche and Paulus, 2000, 2001; Wassermann and Grafman, 2005). The induction of long-term potentiation/ depression-like plasticity by tDCS is implicated by blockade of the aftereffects using NMDA receptor antagonists (Liebetanz et al., 2002; Nitsche et al., 2003a, 2004a,b; Poreisz et al., 2007). tDCS studies of the PFC have measured cognitive functions such as working memory (Fregni et al., 2005; Marshall et al., 2005; Boggio et al., 2006) and learning (Kincses et al., 2004; Flöel et al., 2008). The DLPFC is the most crucial site for dopaminergic effects on cognitive functions (Braver and Cohen, 2000; Cools et al., 2002), which are associated with endogenous DA release (Phillips et al., 2004; Aalto et al., 2005; Sawamoto et al., 2008). Dopamine has a neuroplasticity-modifying influence on the effects of tDCS (Kuo et al., 2008).

Following a simple mechanistic approach, for cortical areas critically involved in task performance, a tDCS-induced increase of excitability could exert behavioral improvements, just as decreased excitability could diminish performance. However, tDCS-induced changes in excitability or tDCS application preceding learning processes have been shown to cause inverse or preventative effects on proceeding manipulations of neuronal excitability and synaptic plasticity (Lang et al., 2004; Siebner et al., 2004). Learning phase-specific effects of tDCS were found in a visuomotor task by enhanced performance with anodal tDCS on 
areas V5 and M1 only in the early phase of learning (Antal et al., 2004a), and with cathodal tDCS of V5 when the task was overlearned (Antal et al., 2004b).

We aimed to evaluate the influence of tDCS on frontal lobe function by use of the TOL test. We hypothesized that dependent on the preceding learning processes, anodal and cathodal tDCS exert differential influences on TOL performance, with the effects apparent specifically at high levels of task load.

\section{Materials and Methods}

Participants. Twenty-four right-handed healthy subjects ( 19 women; average age $24 \pm 3.16 \mathrm{SD}$; average years of education $16.8 \pm 2.63 \mathrm{SD}$ ) between 19 and 32 years of age participated in this study. All subjects had no previous history of neurological and/or psychiatric disorder and were not taking any medication. They were naive for the aim of the study and all participants provided informed consent. Approval by the local ethics committee was obtained before commencement of the study, which was conducted in strict accordance with the local ethics policies.

Experimental design (Fig. 1). The study was designed as a single blind within-subject, sham-controlled randomized cross-over trial. The participants underwent a training period for the TOL for each of the three sessions. The participants solved 10 sets (two problems at five levels of difficulty) of TOL problems in the practice session and 35 problems for each of two blocks of the test session. The tDCS stimulation for the test session started 5 min before the TOL test began to assure that the cognitive testing started under the stimulatory condition. The second TOL block was performed immediately after tDCS. The second and third sessions took place after a 1 week intersession interval. Session order by stimulation condition was counterbalanced across participants.

The participants were instructed to imagine the moves necessary to solve the task and to indicate the total number of moves by pressing the mouse button corresponding to the correct number on the computer monitor. The actual planning was insured by monitoring a change in "computing" time that increased linearly with level of task demand. The order of TOL problems was alternated, and the arrangement of the answer choices presented on the display was randomized between sessions to prevent serial learning. As a discriminating index for the possible subtle functional affects of tDCS on planning function, task load scaled five levels; however the levels were collapsed into two levels for analysis, thereby making clearly definable categories of low-load ("easy") versus high-load ("difficult") problems.

To further investigate the stability of the learned skill, we retested participants after $6(n=9)$ and $12(n=10)$ months under sham tDCS. We aimed to assess whether the results represented an acute effect on task performance or a long-lasting effect at the level of cognitive skill learning.

Transcranial direct current stimulation. tDCS was administered over a 15 min period using a battery-driven, constant-current stimulator (Rolf Schneider Electronic) with a $1 \mathrm{~mA}$ current run between a pair of watersoaked sponge electrodes $\left(35 \mathrm{~cm}^{2}\right.$ surface). The electrodes were fixed over F3 [International 10-20 system of electrode placement (Jasper, 1958)] and contralaterally above the right orbit. Anodal stimulation (Nitsche and Paulus 2001) was performed with the anode placed above F3 and the cathode above the contralateral orbita. The montage was reversed for cathodal tDCS (Nitsche et al., 2003c). Sham stimulation was conducted with $5 \mathrm{~s}$ of tDCS applied at the onset, after which the DC stimulator was deramped for $5 \mathrm{~s}$ (Siebner et al., 2004; Lang et al., 2007; Nitsche et al., 2007a).

Cognitive testing. Cognitive planning skills were assessed using the
Tower of London Test rendered using e-Prime version 1.1 software (Psychology Software Tools), administered on a personal computer. For the TOL puzzle, the subject must achieve a specified final configuration with the rendering of planned moves, and therefore must anticipate the consequences of each action taken. The dependent variables for this test are reaction time (RT) and accuracy (ACC), which comprises the number of matches of the correct number of moves needed to reach the goal configuration in a specific time period (30 s). The independent variables were stimulation condition, block, and task load.

Data analysis. For each subject, the mean reaction time and accuracy [percentage correct $=$ (number of correct solutions/total number of trials) $\times 100$ ] were calculated and entered into a repeated-measures ANOVA (rmANOVA) with the factors condition cathodal,anodal,sham $_{\text {, }}$ (three levels), block during,after (two levels; during and after tDCS), and

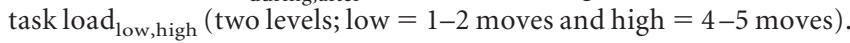
Due to the cross-over design, the effects of repeated performance sessions had to be tested separately adopting a second univariate ANOVA with the factor session ${ }_{1-3}$ instead of condition cathodal,anodal,sham $_{\text {. }}$

To detect learning-phase-dependent effects (factor session ${ }_{1-3}$, three levels), separate one-way ANOVAs were performed on mean performance values for each of the three stimulation conditions. The effect sizes (Cohen's $d$ ) of anodal and cathodal tDCS compared with sham stimulation were computed. To allow for a direct comparison of anodal and cathodal stimulation effects at the different learning phases (sessions), an

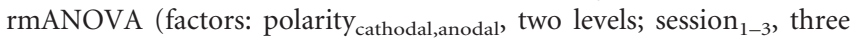
levels) was performed on data normalized by the respective session for sham-performance values (percentage change of stimulation condition from sham performance).

To further assess the specific effect of stimulation order, comparisons were made between performance when sham preceded real (cathodal and anodal) $\mathrm{tDCS}\left(\mathrm{tDCS}_{\mathrm{SR}}\right)$ and the results from when real was applied before sham $\left(\mathrm{tDCS}_{\mathrm{RS}}\right)$. Then, a second rmANOVA was used to test for an effect of order of both real tDCS groups: anodal tDCS before cathodal $\left(\mathrm{tDCS}_{\mathrm{AC}}\right)$ and cathodal before anodal $\left(\mathrm{tDCS}_{\mathrm{CA}}\right)$. Additionally, paired $t$ tests were used to test for a difference between the two retest intervals ( 6 or 12 months) and for a difference between session 3 and the retest session.

When appropriate, a Greenhouse-Geisser correction for degrees of freedom was used. Results were considered significant if the $p$ value was $\leq 0.05$. When an interaction $F$ value was significant, follow-up ANOVAs 

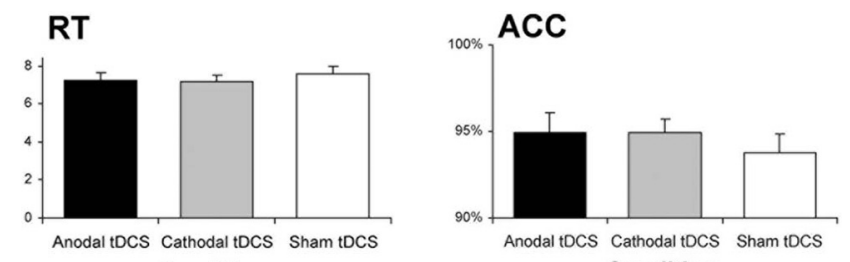

Condition
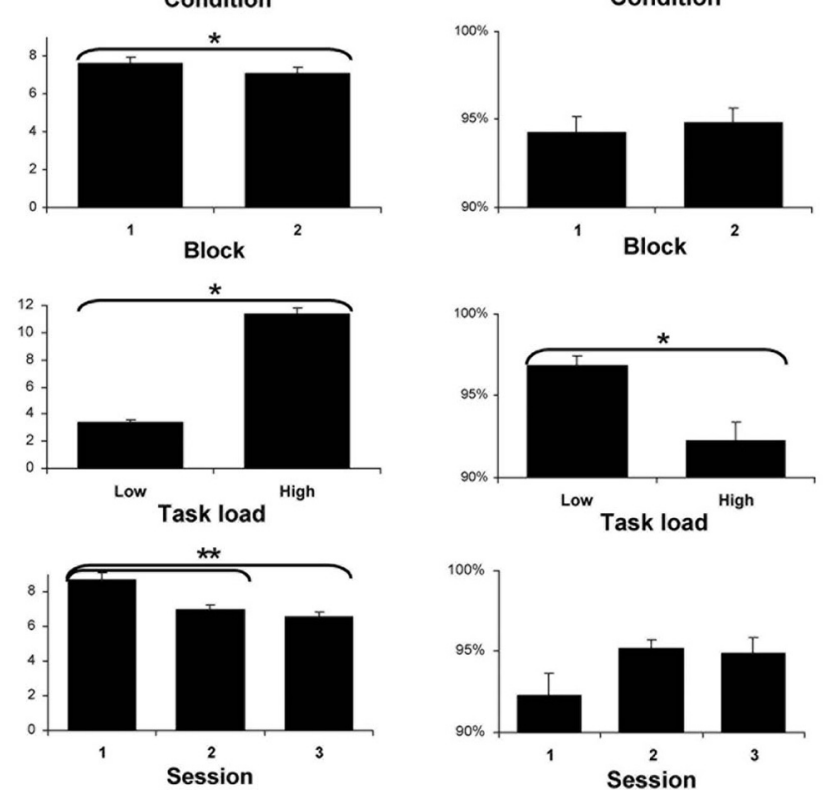

Figure 2. The mean reaction times (seconds) and the percentage of accurate responses for the TOL task for each condition, across all sessions, including both blocks and task load levels (1to 2- or 4- to 5-move problems). The order of stimulation conditions was counterbalanced across participants. Error bars indicate \pm SEM.

and/or post hoc paired-sample $t$ tests (two-tailed) were performed with a Bonferroni correction when appropriate.

Data were analyzed using SPSS 15.0. All figures represent group data. Error bars refer to the SEM.

\section{Results}

None of the participants experienced adverse side effects. One participant dropped out before completion of the third session due to concerns about the stimulation. Subjects undergoing sham tDCS reported a tingling sensation, as for the real tDCS conditions.

\section{General effects (Fig. 2)}

Reaction time

The rmANOVA for reaction time showed a significant effect of block $\left(F_{(1,23)}=22.018, p<0.001\right)$ and task load $\left(F_{(1,23)}=\right.$ $429.588, p<0.001)$, indicating a shorter reaction time during the second performance block after stimulation and an increase of reaction time with the higher load. A significant task load $\times$ block interaction $\left(F_{(1,23)}=22.816, p<0.001\right)$ was found with a posttDCS block decrease in RT for high task load only $\left(F_{(1,23)}=\right.$ 24.680, $p<0.001$ ), most likely representing within-session learning of difficult problems. There was no main effect of stimulation condition $\left(F_{(2,46)}=0.614, p=0.55\right)$ and no further interactions. An rmANOVA showed a significant effect of session $\left(F_{(2,40)}=\right.$ 41.062, $p<0.001$ ), where all sessions significantly differed with an average RT of $8.73 \pm 0.336$ for session $1,7.137 \pm 0.287$ for session 2 , and $6.612 \pm 0.317$ for session 3 .
Accuracy

The overall accuracy rate was $94.4 \%$. Here, the rmANOVA also showed a main effect of task load $\left(F_{(1,23)}=19.648, p<0.001\right)$ but not of block $\left(F_{(1,22)}=0.651 p=0.428\right)$. An interaction of stimulation condition $\times$ task load $\left(F_{(2,46)}=5.410, p=0.008\right)$ was found. Post hoc analysis revealed decreased ACC with the high task load for the cathodal $\left(F_{(1,21)}=7.016, p=0.015\right)$ and sham $\left(F_{(1,21)}=22.501, p<0.001\right)$ but not in the anodal tDCS group $\left(F_{(1,21)}=2.980, p=0.099\right)$, indicating that anodal tDCS appears to mitigate the task-load-dependent performance deterioration. No overall effect of stimulation condition $\left(F_{(2,46)}=0.645, p=\right.$ $0.529)$ and no further interactions were observed. An rmANOVA showed a significant effect of session $\left(F_{(2,40)}=3.690, p=0.034\right)$, with a trend toward improvement from session 1 to session 2 $(p=0.062)$ and significantly improved accuracy from session 1 to session $3(p=0.028)$.

Polarity-specific effects are learning phase dependent (Fig. 3) To test for differential effects of stimulation conditions on acrosssession learning, three separate rmANOVA tests for each condition were performed. For reaction time, anodal tDCS showed a significant effect of $\operatorname{session}_{1-3}\left(F_{(2,20)}=11.402, p<0.001\right)$. Post hoc $t$ tests showed improvements of reaction time from session 1 to session $2(p=0.009)$ and from session 1 to session $3(p<$ 0.001 ) and borderline difference from session 2 to session 3 ( $p=$ 0.071 ), representative of a learning effect by decreased reaction times. Cathodal tDCS yielded no significant effect of session s-3 $_{1-3}$ $\left(F_{(2,21)}=0.596, p=0.560\right)$, while sham tDCS showed a trend toward significant difference $\left(F_{(2,21)}=2.565, p=0.101\right)$.

For accuracy, no effect of session ${ }_{1-3}$ was found for the anodal $\left(F_{(1,21)}=2.519, p=0.105\right)$, cathodal $\left(F_{(1,21)}=1.694, p=0.208\right)$, or sham $\left(F_{(1,21)}=1.901, p=0.174\right)$ tDCS groups.

Calculation of effect sizes (Cohen's $d$ ) revealed medium and medium-to-large effects $(0.5-0.8)$ of stimulation on reaction time and accuracy comparing cathodal with sham in the first and anodal with sham stimulation in the third session (Fig. 3, Table 1).

Direct comparison of normalized data showed significant interactions of polarity anodal,cathodal $\times \operatorname{session}_{1-3}\left(\mathrm{RT}: F_{(2,21)}=\right.$ 4.813, $p=0.03$; ACC: $F_{(2,21)}=3.337, p=0.055$, respectively). Post hoc analysis revealed an effect of polarity on reaction time for session $1\left(F_{(1,7)}=13.107, p=0.009\right)$ in which cathodal tDCS yielded a higher relative improvement, therefore faster RT, compared with sham tDCS than anodal tDCS. No significant differences were found for session 2. For session 3 both RT $\left(F_{(1,7)}=\right.$ 5.886, $p=0.046)$ and $\operatorname{ACC}\left(F_{(1,7)}=7.012, p=0.033\right)$ post hoc tests revealed higher improvement of anodal tDCS normalized to sham compared with the normalized cathodal tDCS data (Fig. 4).

\section{Stimulation sequence predicts performance gains}

The order of sham tDCS application relative to real (cathodal or anodal) tDCS showed no effect on $\operatorname{RT}\left(F_{(1,20)}=0.074, p=0.788\right)$ $\left(\mathrm{tDCS}_{\mathrm{SR}}: 7.57 \pm 0.46, \mathrm{tDCS}_{\mathrm{RS}}: 7.42 \pm 0.32\right)$ and $\mathrm{ACC}\left(F_{(1,19)}=\right.$ $1.452, p=0.243)\left(\mathrm{tDCS}_{\mathrm{SR}}: 0.93 \pm 0.01, \mathrm{tDCS}_{\mathrm{RS}}: 0.95 \pm 0.01\right)$, indicating that order of sham tDCS relative to real tDCS was ineffectual on learning. The comparison of order of both real tDCS groups (cathodal and anodal tDCS: sequence $\mathrm{CA}_{\mathrm{AC}}$ ) revealed a highly significant group effect of sequence ${ }_{\mathrm{CA}, \mathrm{AC}}\left(F_{(1,22)}=\right.$ 8.935, $p=0.007)$ with decreased reaction time for $\mathrm{tDCS}_{\mathrm{CA}}$ $(6.35 \pm 0.35 \mathrm{~s})$ compared with $\mathrm{tDCS}_{\mathrm{AC}}(7.91 \pm 0.38 \mathrm{~s})$. Accuracy data showed a borderline effect $\left(F_{(1,22)}=3.494, p=0.075\right)$, for which $\mathrm{tDCS}_{\mathrm{CA}}$ showed a trend for better performance than the $\mathrm{tDCS}_{\mathrm{AC}}$. A significant interaction of task load high,low $\times$ sequence $_{\mathrm{CA} / \mathrm{AC}}\left(F_{(1,22)}=7.749, p=0.011\right)$ showed faster reaction 
time by $\mathrm{tDCS}_{\mathrm{CA}}$ for both task load levels with a distinct advantage for $\mathrm{tDCS}_{\mathrm{AC}}$ at high task loads. The mean reaction times for the low load are as follows: $\mathrm{tDCS}_{\mathrm{CA}}$ : $3.09 \pm 0.20 \mathrm{~s}, \mathrm{tDCS}_{\mathrm{AC}}: 3.73 \pm 0.21 \mathrm{~s}$; and for the high load: $\mathrm{tDCS}_{\mathrm{CA}}: 10.17 \pm 0.56 \mathrm{~s}$, $\mathrm{tDCS}_{\mathrm{AC}}: 12.69 \pm 0.61 \mathrm{~s}$.

Stimulation modulated performance gains are persistent

We aimed to address whether the short term effects on planning ability induced by tDCS could translate into long-term gains. Participants performed the same for RT $\left(F_{(1,16)}=0.103, p=0.752\right)$ and ACC $\left(F_{(1,16)}=0.681, p=0.421\right)$ regardless of the length of interval of testing ( 6 or 12 months). Notably, the performance was maintained from session 3 to the retest session for RT $\left(F_{(1,16)}=0.011, p=0.918\right)$ and ACC $\left(F_{(1,16)}=1.661, p=0.216\right)$. In the retest session, stimulation sequence had no effect on accuracy $\left(F_{(1,16)}=0.092, p=\right.$ $0.765)$; however, a significant effect was found for reaction time $\left(F_{(1,16)}=17.357\right.$, $p=0.001)$ by which the $\mathrm{tDCS}_{\mathrm{CA}}$, even a year after exposure, yielded a $42 \%$ faster RT than $\mathrm{tDCS}_{\mathrm{AC}}$.

For a comprehensive overview of the results, see supplemental material 1 and 2 (available at www.jneurosci.org).

\section{Discussion}

The central and unique findings of this study are that both anodal and cathodal tDCS enhance planning ability as quantified by TOL task performance and that, although not initially hypothesized, these effects are learning phase dependent. Unlike the negative findings from studies using transcranial magnetic stimulation over left DLPFC on TOL performance (Loo et al., 1999; Roth et al., 2004), in our study only $15 \mathrm{~min}$ of $1 \mathrm{~mA}$ of real tDCS led to altered RT and ACC. The polarity-specific effects were more pronounced at the higher task load level, and sustained for up to a year without further tDCS application. To our knowledge this is the first study comparing long- and short-term effects of tDCS of the DLPFC on TOL planning ability and cognitive skill learning. We cannot overlook, however, that gender differences have been found in TOL BOLD activation patterns in which females showed greater recruitment of the DLPFC than males (Boghi et al., 2006), and since our study had a larger proportion of women to men, the factor of gender on how tDCS may cause differential effects on performance should not be dismissed.

In consideration of the learning effects, our study design did not include baseline TOL measurements, potentially masking tDCS effects specific for the early learning phase. To test for polarity-specific across-session effects, a balanced cross-over design was required. With this approach, the sample size of 24 allowed for the random assignment of eight subjects to each stimulation condition at each of the three sessions. However, the mutual dependency of session and condition in this design re-
Table 1. Cohen's $d$

\begin{tabular}{llrlrr}
\hline \multirow{2}{*}{ Session } & \multicolumn{2}{l}{ Reaction time } & & & \multicolumn{2}{l}{ TOL accuracy } & \\
\cline { 2 - 3 } \cline { 6 - 6 } & Anodal & Cathodal & & Anodal & Cathodal \\
\hline 1 & -0.216 & 0.458 & & -0.115 & 0.844 \\
2 & 0.120 & 0.378 & & 0.377 & 0.216 \\
3 & 0.672 & -0.231 & & 0.484 & -0.445 \\
\hline
\end{tabular}

Effect sizes according to Cohen's $d\left(d=M_{1}-M_{2} / S D_{\text {pooled; }} M\right.$, mean) reported for the normalized mean gain reaction time and accuracy data for both real tDCS groups according to session. The effect size conventions are as follows: small, $d=0.2$; medium, $d=0.5$; and large, $d=0.8$.

quired a multilevel statistical approach including the calculation of effect size and normalization of data.

While many TOL as well as tDCS intervention studies have evaluated cognitive performance for one session, our results show differential changes in tDCS effects on planning time and accuracy over consecutive sessions. RT appears to serve as an appropriate index of planning ability, as participants were instructed to mentally solve the problem and RT increased linearly with increased task load. Participants become more proficient beyond general problem solving ability after several repetitions of TOL problems (Ouellet et al., 2004). The learning effect on planning performance in the TOL task (Beauchamp et al., 2003) could 


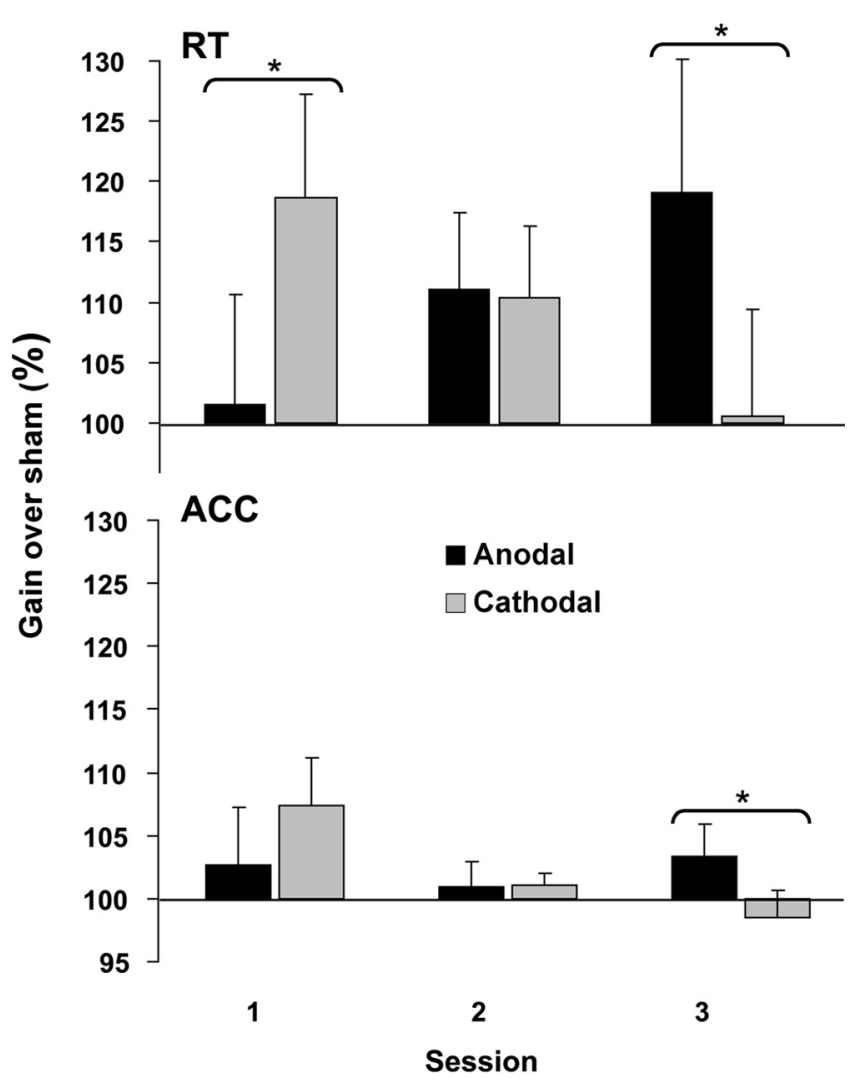

Figure 4. Normalized average TOL performance gain with anodal (black bars) and cathodal (gray bars) tDCS of the left dorsolateral prefrontal cortex (DLPFC). Normalized values were obtained setting the performance during each of the corresponding sham tDCS sessions to 100 . Session-specific significant differences in reaction time and accuracy for planning performance of anodal DLPFC compared with cathodal DLPFC stimulation were found. Error bars indicate \pm SEM.

be due not only to better encoding of problems and increased task-related working memory capacity (Garavan et al., 2000), but also to the efficient development of specific sets of rules and strategies to perform this cognitive skill.

The benefits of tDCS on planning performance are rendered by applying a particular polarity at a specific learning phase. As depicted in Figure 4, the facilitating effect of cathodal tDCS during acquisition/early learning, as opposed to the facilitating effect of anodal tDCS on RT under late learning reveals a learningphase-dependent dissociation of stimulation effects. Moreover, the amelioration of learning-phase differences by minimizing the learning curve is established by cathodal tDCS. Cathodal tDCS improved performance at an early learning phase when participants perform the worst at the task (Fig. 3). Anodal tDCS boosted performance under late learning when participants have characteristically already become proficient.

In healthy participants, blood flow increases in the DLPFC in early stages of performance, while decreased neuronal activity with late learning has been associated with increased neural efficiency as TOL performance improves (Beauchamp et al., 2003). Most likely, recruitment of the left DLPFC and associate areas in the frontostriatal loop enhance the ability to adapt and generate efficient strategies (Cazalis et al., 2003), via increased excitability, which strengthens neuronal synaptic connections and makes them more efficacious (Nitsche et al., 2003b). When learning is paired with tDCS, however, the interaction of the excitability inherent to performing novel tasks in addition to tDCS induced excitability results in distinct differences depending on the phase of learning (i.e., early and late). These differences are conceivably related to dopamine levels, which appear to have dosedependent, task-specific effects on performance (Kulisevsky, 2000) and possibly on changes in the participation of the dorsolateral prefrontal network in task performance.

Unlike findings with tDCS of the motor cortex, in which increased excitability by anodal tDCS enhanced motor performance, while cathodal tDCS reduced improvement in skill acquisition (Vines et al., 2006), in our study the behavioral effects of the excitability changes seem to depend on the preexisting state of the cortex, as subject to previous experience. Accordingly, results from $\mathrm{tDCS}$ on visuomotor learning indicate a sensitivity to anodal excitability enhancement during the early phase of learning, while conversely when the optimal movement is learned, a cathodal tDCS-generated increase of the signal-to-noise ratio by suppression of cortical activity might improve performance (Antal et al., 2004a,b). Otherwise, for TOL performance, a single optimal reaction has to be formed. This may be grounds for why improvement of the signal-to-noise ratio through cathodal tDCS would benefit early performance. Once established, in later sessions excitability increases by anodal tDCS might reactivate those already-shaped experience-dependent responses and not alternative ones.

While with our design we cannot disregard the possibility that right frontopolar stimulation plays a role in the effects, a functional distinction between the frontopolar and the dorsolateral cortices contributes to a hierarchical system specialized for the functions (Christoff and Gabrieli 2000) involved in Tower of London performance, and studies suggest a specific role of left frontal areas in these functions (Gouveia et al., 2007). An experiment targeting the right DLPFC (F4) and the left frontopolar cortex above the left orbit will further clarify their respective contributions to the results. The results of this experiment will be reported in a forthcoming manuscript.

In the present study, cathodal tDCS appears to act as a neuronal noise reducer or filter, facilitating acquisition of the executive functions related to TOL performance. In studies on motor cortex function, this focalizing effect has been proposed to be mediated via dopamine and its role in NMDA receptor-dependent neuroplasticity (Nitsche et al., 2003b, 2006; Kuo et al., 2008), whereby information processing may be enhanced by an increased signal-to-noise ratio (Frank and O'Reilly, 2006). Cathodal tDCS may be particularly beneficial when paired with the inherent activation that occurs with acquisition of a new task or exposure to new conditions, in which the TOL task induces noisy activations in the initial learning phase, which then stabilize in later phases.

Alternatively, anodal tDCS appears to provide benefits under already-trained conditions, but not during exposure to a novel task. Anodal tDCS was found to have reverse effects, inhibiting motor cortex excitability, with L-dopa administration (Kuo et al., 2008). This indicates that while the effects of $\mathrm{tDCS}$ require a physiological concentration of DA, a reversal of effects on excitability can occur depending on the DA level. Extremely high or low DA concentrations, for example due to periods of stress, can alter the DLPFC network balance to incommensurate inhibitory interneuron activation (Kröner et al., 2007). Anodal tDCS resulted in higher accuracy and faster reaction times in later sessions, presumably via increased neural excitability under more automatic learning conditions when DA levels have receded. This model coincides with homeostatic plasticity whereby low background activity (e.g., pretreatment with cathodal tDCS in an ear- 
lier session) would enhance the facilitatory associative plasticity related to learning (Nitsche et al., 2007b), whereas high excitability (e.g., anodal tDCS) would inhibit it; likewise the strength of this homeostatic effect would diminish as learning reached asymptotic levels.

The analysis of general effects suggests a differential task loadspecific effect of stimulation by which the detrimental effect of high task load on accuracy observed for the cathodal and sham tDCS groups was not apparent for the anodal tDCS group, indicating amelioration by performance gain. This supports our hypothesis that the affect of anodal tDCS is apparent at higher levels of task load, as only difficult problems reflect high-level cognitive function. Most likely, this finding does not reflect boosted procedural learning alone, such as the motor learning described by Nitsche et al. (2003b), since the effects are specific to higher task demand favoring improvements on cognitive skill learning or working memory associated with this task.

The results from the retest experiment clearly show that these phase- and polarity-specific beneficial effects of the optimal tDCS sequence, being cathodal tDCS preceding anodal tDCS, persist well beyond the acute application, presumably, as a function of learning mechanisms and long-term cognitive effects of tDCS.

These data demonstrate the viability of using tDCS to influence the circuitry involved in planning performance and cognitive skill learning and support a critical role of the DLPFC in solving TOL problems and the persistence of the learned skill over time. These data suggest that the appropriate tDCS protocol may facilitate the acquisition and long-term retention of specific planning abilities, opening new perspectives for the treatment of patients with frontal lobe pathology and individuals with learning disorders. tDCS studies are under way to test these findings prospectively and to investigate the functional lateralization of the DLPFC on executive function.

\section{References}

Aalto S, Brück A, Laine M, Någren K, Rinne JO (2005) Frontal and temporal dopamine release during working memory and attention tasks in healthy humans: a positron emission tomography study using the high-affinity dopamine $\mathrm{D}_{2}$ receptor ligand $\left[{ }^{11} \mathrm{C}\right] \mathrm{FLB}$ 457. J Neurosci 25:2471-2477.

Antal A, Nitsche MA, Kincses TZ, Kruse W, Hoffmann K-P, Paulus W (2004a) Facilitation of visuo-motor learning by transcranial direct current stimulation of the motor and extrastriate visual areas in humans. Eur J Neurosci 19:2888-2892.

Antal A, Nitsche MA, Kruse W, Kincses TZ, Hoffmann K-P, Paulus W (2004b) Direct current stimulation over V5 enhances visuomotor coordination by improving motion perception in humans. J Cogn Neurosci 16:521-527.

Baker SC, Rogers RD, Owen AM, Frith CD, Dolan RJ, Frackowiak RSJ, Robbins TW (1996) Neural systems engaged by planning: a PET study of the Tower of London task. Neuropsychologia 34:515-526.

Beauchamp MH, Dagher A, Aston JA, Doyon J (2003) Dynamic functional changes associated with cognitive skill learning of an adapted version of the Tower of London task. Neuroimage 20:1649-1660.

Boggio PS, Ferrucci R, Rigonatti SP, Covre P, Nitsche M, Pascual-Leone A, Fregni F (2006) Effects of transcranial direct current stimulation on working memory in patients with Parkinson's disease. J Neurol Sci 249:31-38.

Boghi A, Rasetti R, Avidano F, Manzone C, Orsi L, D’Agata F, Caroppo P, Bergui M, Rocca P, Pulvirenti L, Bradac GB, Bogetto F, Mutani R, Mortara $\mathrm{P}$ (2006) The effect of gender on planning: an fMRI study using the Tower of London task. Neuroimage 33:999-1010.

Braver TS, Cohen JD (2000) On the control of control: the role of dopamine in regulating prefrontal function and working memory. In: Attention and performance, Vol XVIII, Control of cognitive processes (Monsell S, Driver J, eds), pp 713-737. Cambridge, MA: MIT.

Cazalis F, Valabrègue R, Pélégrini-Issac M, Asloun S, Robbins TW, Granon S (2003) Individual differences in prefrontal cortical activation on the
Tower of London planning task: implication for effortful processing. Eur J Neurosci 17:2219-2225.

Cazalis F, Feydy A, Valabrègue R, Pélégrini-Issac M, Pierot L, Azouvi P (2006) fMRI study of problem-solving after severe traumatic brain injury. Brain Inj 20:1019-1028.

Christoff K, Gabrieli JDE (2000) The frontopolar cortex and human cognition: evidence for a rostrocaudal hierarchical organization within the human prefrontal cortex. Psychobiology 28:168-186.

Cools R, Stefanova E, Barker RA, Robbins TW, Owen AM (2002) Dopaminergic modulation of high-level cognition in Parkinson's disease: the role of the prefrontal cortex revealed by PET. Brain 125:584-594.

Dagher A, Owen AM, Boecker H, Brooks DJ (1999) Mapping the network for planning: a correlation PET activation study with the Tower of London task. Brain 122:1973-1987.

Flöel A, Rösser N, Michka O, Knecht S, Breitenstein C (2008) Noninvasive brain stimulation improves language learning. J Cogn Neurosci 20:1415-1422.

Frank MJ, O’Reilly RC (2006) A mechanistic account of striatal dopamine function in human cognition: psychopharmacological studies with cabergoline and haloperidol. Behav Neurosci 120:497-517.

Fregni F, Boggio PS, Nitsche M, Bermpohl F, Antal A, Feredoes E, Marcolin MA, Rigonatti SP, Silva MT, Paulus W, Pascual-Leone A (2005) Anodal transcranial direct current stimulation of prefrontal cortex enhances working memory. Exp Brain Res 166:23-30.

Garavan H, Kelley D, Rosen A, Rao SM, Stein EA (2000) Practice-related functional activation changes in a working memory task. Microsc Res Tech 51:54-63.

Gouveia PA, Brucki SM, Malheiros SM, Bueno OF (2007) Disorders in planning and strategy application in frontal lobe lesion patients. Brain Cogn 63:240-246.

Jasper HH (1958) The ten-twenty electrode system of the International Federation. Electroencephalogr Clin Neurophysiol 10:371-375.

Kincses TZ, Antal A, Nitsche MA, Bártfai O, Paulus W (2004) Facilitation of probabilistic classification learning by transcranial direct current stimulation of the prefrontal cortex in the human. Neuropsychologia 42:113-117.

Kröner S, Krimer LS, Lewis DA, Barrionuevo G (2007) Dopamine increases inhibition in the monkey dorsolateral prefrontal cortex through cell typespecific modulation of interneurons. Cereb Cortex 17:1020-1032.

Kulisevsky J (2000) Role of dopamine in learning and memory: implications for the treatment of cognitive dysfunction in patients with Parkinson's disease. Drugs Aging 16:365-379.

Kuo MF, Paulus W, Nitsche MA (2008) Boosting focally-induced brain plasticity by dopamine. Cereb Cortex 18:648-651.

Lang N, Siebner HR, Ernst D, Nitsche MA, Paulus W, Lemon RN, Rothwell JC (2004) Preconditioning with transcranial direct current stimulation sensitizes the motor cortex to rapid-rate transcranial magnetic stimulation and controls the direction of after-effects. Biol Psychiatry 56:634-639.

Lang N, Siebner HR, Chadaide Z, Boros K, Nitsche MA, Rothwell JC, Paulus W, Antal A (2007) Bidirectional modulation of primary visual cortex excitability: a combined tDCS and rTMS study. Invest Ophthalmol Vis Sci 48:5782-5787.

Lazeron RH, Rombouts SA, Scheltens P, Polman CH, Barkhof F (2004) An fMRI study of planning-related brain activity in patients with moderately advanced multiple sclerosis. Mult Scler 10:549-555.

Liebetanz D, Nitsche MA, Tergau F, Paulus W (2002) Pharmacological approach to the mechanisms of transcranial DC-stimulation-induced aftereffects of human motor cortex excitability. Brain 125:2238-2247.

Loo C, Mitchell P, Sachdev P, McDarmont B, Parker G, Gandevia S (1999) Double-blind controlled investigation of transcranial magnetic stimulation for the treatment of resistant major depression. Am J Psychiatry 156:946-948.

Marshall L, Mölle M, Siebner HR, Born J (2005) Bifrontal transcranial direct current stimulation slows reaction time in a working memory task. BMC Neurosci 6:23.

Morris RG, Ahmed S, Syed GM, Toone BK (1993) Neural correlates of planning ability: frontal lobe activation during the Tower of London test. Neuropsychologia 31:1367-1378.

Nitsche MA, Paulus W (2000) Excitability changes induced in the human motor cortex by weak transcranial direct current stimulation. J Physiol 527:633-639.

Nitsche MA, Paulus W (2001) Sustained excitability elevations induced by 
transcranial DC motor cortex stimulation in humans. Neurology 57:1899-1901.

Nitsche MA, Fricke K, Henschke U, Schlitterlau A, Liebetanz D, Lang N, Henning S, Tergau F, Paulus W (2003a) Pharmacological modulation of cortical excitability shifts induced by transcranial direct current stimulation in humans. J Physiol 553:293-301.

Nitsche MA, Schauenburg A, Lang N, Liebetanz D, Exner C, Paulus W, Tergau F (2003b) Facilitation of implicit motor learning by weak transcranial direct current stimulation of the primary motor cortex in the human. J Cogn Neurosci 15:619-626.

Nitsche MA, Nitsche MS, Klein CC, Tergau F, Rothwell JC, Paulus W (2003c) Level of action of cathodal DC polarisation induced inhibition of the human motor cortex. Clin Neurophysiol 114:600-604.

Nitsche MA, Jaussi W, Liebetanz D, Lang N, Tergau F, Paulus W (2004a) Consolidation of externally induced human motor cortical neuroplasticity by d-cycloserine. Neuropsychopharmacology 29:1573-1578.

Nitsche MA, Liebetanz D, Schlitterlau A, Henschke U, Fricke K, Frommann K, Lang N, Henning S, Paulus W, Tergau F (2004b) GABAergic modulation of DC-stimulation-induced motor cortex excitability shifts in the human. Eur J Neurosci 19:2720-2726.

Nitsche MA, Lampe C, Antal A, Liebetanz D, Lang N, Tergau F, Paulus W (2006) Dopaminergic modulation of long-lasting direct current-induced cortical excitability changes in the human motor cortex. Eur J Neurosci 23:1651-1657.

Nitsche MA, Doemkes S, Karaköse T, Antal A, Liebetanz D, Lang N, Tergau F, Paulus W (2007a) Shaping the effects of transcranial direct current stimulation of the human motor cortex. J Neurophysiol 97:3109-3117.

Nitsche MA, Roth A, Kuo MF, Fischer AK, Liebetanz D, Lang N, Tergau F, Paulus W (2007b) Timing-dependent modulation of associative plasticity by general network excitability in the human motor cortex. J Neurosci 27:3807-3812.

Olesen PJ, Westerberg H, Klingberg T (2004) Increased prefrontal and parietal activity after training of working memory. Nat Neurosci 7:75-79.

Ouellet MC, Beauchamp MH, Owen AM, Doyon J (2004) Acquiring a cognitive skill with a new repeating version of the Tower of London task. Can J Exp Psychol 58:272-288.

Owen AM (1997) Cognitive planning in humans: neuropsychological, neuroanatomical and neuropharmacological perspectives. Prog Neurobiol 53:431-450.

Owen AM, Doyon J, Petrides M, Evans AC (1996) Planning and spatial working memory: a positron emission tomography study in humans. Eur J Neurosci 8:353-364.

Peretti CS, Danion JM, Gierski F, Grangé D (2002) Cognitive skill learning and aging: a component process analysis. Arch Clin Neuropsychol 17:445-459.

Phillips AG, Ahn S, Floresco SB (2004) Magnitude of dopamine release in medial prefrontal cortex predicts accuracy of memory on a delayed response task. J Neurosci 24:547-553.

Poreisz C, Boros K, Antal A, Paulus W (2007) Safety aspects of transcranial direct current stimulation concerning healthy subjects and patients. Brain Res Bull 72:208-214.

Rainville C, Amieva H, Lafont S, Dartigues JF, Orgogozo JM, Fabrigoule C (2002) Executive function deficits in patients with dementia of the Alzheimer's type: a study with a Tower of London task. Arch Clin Neuropsychol 17:513-530.

Rasser PE, Johnston P, Lagopoulos J, Ward PB, Schall U, Thienel R, Bender S, Toga AW, Thompson PM (2005) Functional MRI BOLD response to Tower of London performance of first-episode schizophrenia patients using cortical pattern matching. Neuroimage 26:941-951.

Roth HL, Nadeau SE, Triggs WJ (2004) Effect of repetitive transcranial magnetic stimulation on rate of memory acquisition. Neurology 63:1530-1531.

Rowe JB, Owen AM, Johnsrude IS, Passingham RE (2001) Imaging the mental components of a planning task. Neuropsychologia 39:315-327.

Sawamoto N, Piccini P, Hotton G, Pavese N, Thielemans K, Brooks DJ (2008) Cognitive deficits and stratio-frontal dopamine release in Parkinson's disease. Brain 131:1294-1302.

Schall U, Johnston P, Lagopoulos J, Jüptner M, Jentzen W, Thienel R, Dittmann-Balçar A, Bender S, Ward PB (2003) Functional brain maps of Tower of London performance: a PET and fMRI study. Neuroimage 20:1154-1161.

Shallice T (1982) Specific impairments in planning. Philos Trans R Soc Lond B Biol Sci 298:199-209.

Siebner HR, Lang N, Rizzo V, Nitsche MA, Paulus W, Lemon RN, Rothwell JC (2004) Preconditioning of low-frequency repetitive transcranial magnetic stimulation with transcranial direct current stimulation: evidence for homeostatic plasticity in the human motor cortex. J Neurosci 24:3379-3385.

van den Heuvel OA, Groenewegen HJ, Barkhof F, Lazeron RH, van Dyck R, Veltman DJ (2003) Frontostriatal system in planning complexity: a parametric functional magnetic resonance version of Tower of London task. Neuroimage 18:367-374.

van den Heuvel OA, Veltman DJ, Groenewegen HJ, Cath DC, van Balkom AJ, van Hartskamp J, Barkhof F, van Dyck R (2005) Frontal-striatal dysfunction during planning in obsessive-compulsive disorder. Arch Gen Psychiatry 62:301-309.

Vines BW, Nair DG, Schlaug G (2006) Contralateral and ipsilateral motor effects after transcranial direct current stimulation. Neuroreport 17:671-674.

Walter H, Wolf RC, Spitzer M, Vasic N (2007a) Increased left prefrontal activation in patients with unipolar depression: an event-related, parametric, performance-controlled fMRI study. J Affect Disord 101:175-185.

Walter H, Vasic N, Höse A, Spitzer M, Wolf RC (2007b) Working memory dysfunction in schizophrenia compared to healthy controls and patients with depression: evidence from event related fMRI. Neuroimage 35:1551-1561.

Wassermann EM, Grafman J (2005) Recharging cognition with DC brain polarization. Trends Cogn Sci 9:503-505. 